\title{
Mosaic trisomy 12
}

INSERM

\section{Source}

INSERM. (1999). Orphanet: an online rare disease and orphan drug data base. Mosaic trisomy 12. ORPHA:1698

Mosaic trisomy 12 is a rare chromosomal anomaly syndrome, with a highly variable phenotype, principally characterized by developmental or growth delay, short stature, craniofacial dysmorphism (e.g. turricephaly, tall forehead, downslanting palpebral fissures, posteriorly rotated and low set ears, narrow palate), congenital heart defects (e.g. atrial septal defect, patent ductus arteriosus), hypotonia, and pigmentary dysplasia. Scoliosis, hearing loss, facial/body asymmetry, and intellectual disability have also been reported. 